\title{
Strain-Temperature Discrimination Using Multimode Interference in Tapered Fiber
}

\author{
Ricardo M. André, Claudecir R. Biazoli, Susana O. Silva, Manuel B. Marques, \\ Cristiano M. B. Cordeiro, and Orlando Frazão
}

\begin{abstract}
Tapering single-mode-multimode-single-mode structures to enhance sensitivity is proposed and experimentally demonstrated. 50-mm-long coreless multimode fiber sections are spliced between single-mode fibers (SMFs) and tapered. They are characterized in strain, and an increase in strain sensitivity is obtained with taper diameter reduction. Sensitivities as high as $-23.69 \mathrm{pm} / \mu \varepsilon$ for the $15-\mu \mathrm{m}$ taper are attained. Temperature sensitivities also depend on taper diameter. A combination of two different diameter tapered SMF MMF-SMF structures, with cross-sensitivity to strain and temperature, is proposed as a sensing system for the simultaneous measurement of strain and temperature with resolutions of $\pm 5.6 \mu \varepsilon$ and $\pm 1.6{ }^{\circ} \mathrm{C}$, respectively. A good condition number of 3.16 is achieved with this sensing structure.
\end{abstract}

Index Terms-Multimode interference devices, optical fiber sensors, optical fiber tapers, strain and temperature measurement.

\section{INTRODUCTION}

$\mathbf{M}$ ULTIMODE interference (MMI) has been extensively investigated and many applications, namely in integrated optics, have emerged from such concept. Self-imaging of the input light field has led to the development of directional couplers, beam splitters, multiplexers and others [1]. Multimode interference can also be obtained in optical fibers using a step-index multimode fiber (MMF) and can be used for optical communication and sensing [2]. Usually an MMI-based optical fiber device consists of a multimode fiber section spliced between two single-mode fibers (SMF) forming an SMFMMF-SMF structure (SMS) [3]. SMS fiber structures have been employed as many optical devices such as a displacement sensor [4], a fiber lens [5], refractometer sensors [6], bandpass filters [7], and edge filters [8]. Recently, Wang et al. proposed

Manuscript received July 10, 2012; revised September 19, 2012; accepted November 23, 2012. Date of publication December 11, 2012; date of current version January 9, 2013. This work was supported in part by the Brazilian Funding Agencies FINEP and CAPES. The work of R. M. André was supported by Fundaçăo Calouste Gulbenkian under Award "Prémio de Estímulo à Investigaçăo."

R. M. André, S. O. Silva, and M. B. Marques are with INESC Porto Instituto de Engenharia de Sistemas e Computadores do Porto and Departamento de Física e Astronomia da Faculdade de Ciéncias da Universidade do Porto, Porto 4169-007, Portugal (e-mail: rmeloandre@alunos.fc.up.pt; sfsilva@inescporto.pt; mbmarque@fc.up.pt).

C. R. Biazoli and C. M. B. Cordeiro are with the Instituto de Física "Gleb Wataghin," Universidade Estadual de Campinas, Campinas 13.083-859, Brazil (e-mail: claudecirbiazoli@gmail.com; cmbc@ifi.unicamp.br).

O. Frazão is with INESC Porto, Porto 4169-007, Portugal (e-mail: ofrazao@inescporto.pt).

Color versions of one or more of the figures in this letter are available online at http://ieeexplore.ieee.org.

Digital Object Identifier 10.1109/LPT.2012.2230617

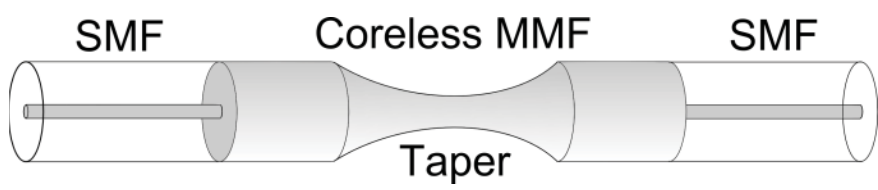

Fig. 1. SMS structure with a taper in the multimode fiber region.

and experimentally demonstrated a high sensitivity evanescent field fiber refractometer based on a tapered multimode-singlemode fiber structure [9].

A tapered SMS structure that works as a multimode interference device is proposed. On the coreless multimode section, tapers with different cross sections ranging from the untapered $125 \mu \mathrm{m}$ to $15 \mu \mathrm{m}$ were fabricated. Different SMS sensing heads with different taper dimensions were characterized in strain and temperature.

\section{SETUP AND Simulations}

The sensing heads are constituted of a section of coreless multimode silica fiber spliced between two single-mode optical fibers (see Fig. 1). On the coreless multimode fiber, a taper is produced by the flame-brushing technique. This technique employs a flame that is swept along the fiber while it is being stretched and thus leads to the reduction of the transverse cross-section and the creation of a taper. The structure will act as a multimode interference device (MMI). In Fig. 2, simulations of the untapered structure (a) and of the structure tapered down to $55 \mu \mathrm{m}$ (b) are presented. The simulation was performed using a Beam Propagation Method (BPM). The parameters used in the simulation are light injected at $1550 \mathrm{~nm}, 50 \mathrm{~mm}$-long section of $125 \mu \mathrm{m}$ coreless fiber with a refractive index of 1.444 and tapered fiber $5 \mathrm{~mm}$ long with $55 \mu \mathrm{m}$-diameter. The transition region was considered to be $12 \mathrm{~mm}$-long and an exponential function. In Fig. 2(a) one can see the usual MMI pattern along the $50 \mathrm{~mm}$ of coreless fiber. In Fig. 2(b), it is evident that even though the taper changes the propagation of light inside the fiber, the MMI pattern is still clearly visible.

Since the self-images resulting from the MMI are more closely packed in the taper region, it is expected that when stretching the tapered SMSs, the shift in the pattern and thus the change in the output light field will be greater. This should in principle lead to much larger strain sensitivity.

\section{EXPERIMENTAL RESULTS}

When analyzing the spectral responses of the several SMS structures with different taper waists, one can identify, for 


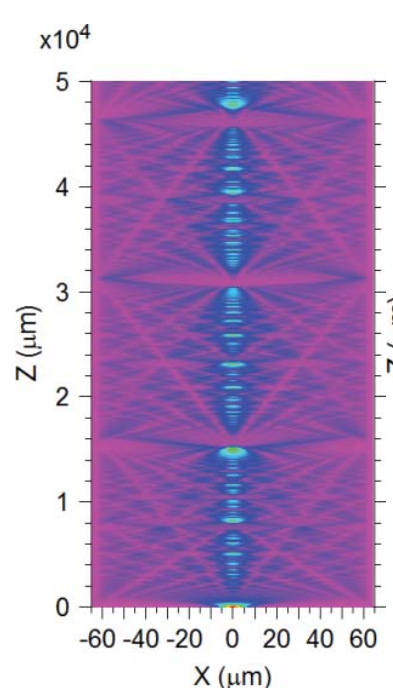

(a)

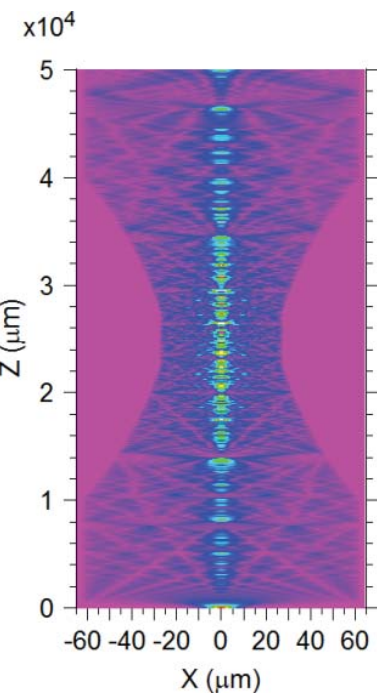

(b)
Fig. 2. BPM calculated electric field amplitude distribution in the $\mathrm{XZ}$ plane for (a) untapered and (b) tapered coreless MMF sandwiched between two standard SMFs. The length of the untapered MMF is $50 \mathrm{~mm}$; the diameter and length of the uniform waist are $55 \mu \mathrm{m}$ and $5 \mathrm{~mm}$, respectively, and the operating wavelength is $1550 \mathrm{~nm}$.

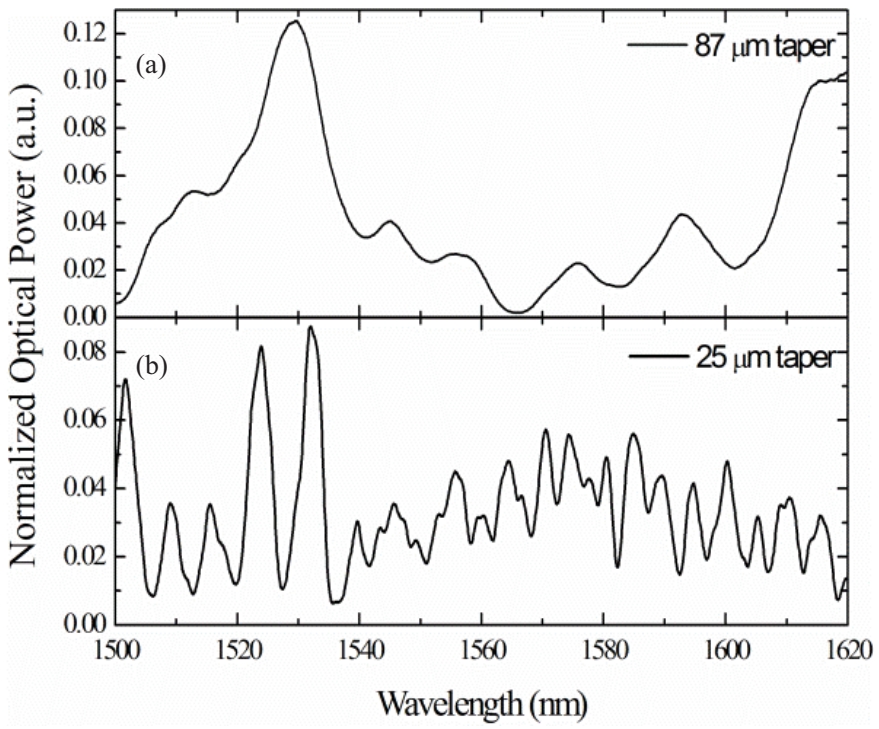

Fig. 3. Spectral responses of (a) $87-\mu \mathrm{m}$-tapered SMS and (b) $25-\mu \mathrm{m}$-tapered SMS.

small diameter reduction (large taper waists), a clear MMI image in the spectral response [see Fig. 3(a)].

As the taper waist is reduced, a smaller number of propagating modes is expected. Going to smaller taper waists $(25 \mu \mathrm{m}$ and $15 \mu \mathrm{m}$ ), the number of modes that propagate through the taper region is much smaller and intermodal interference between just a few modes is present [Fig. 3(b)].

For strain characterization, each SMS structure was placed on micrometric translation stages and subjected to controlled stretching. The total length of the sensing head $(0.4 \mathrm{~m})$ was subjected to strain. To determine the wavelength variations, the wavelength change of the main peaks was monitored through an optical spectrum analyzer with a maximum resolution of $0.05 \mathrm{~nm}$ (see Fig. 4).

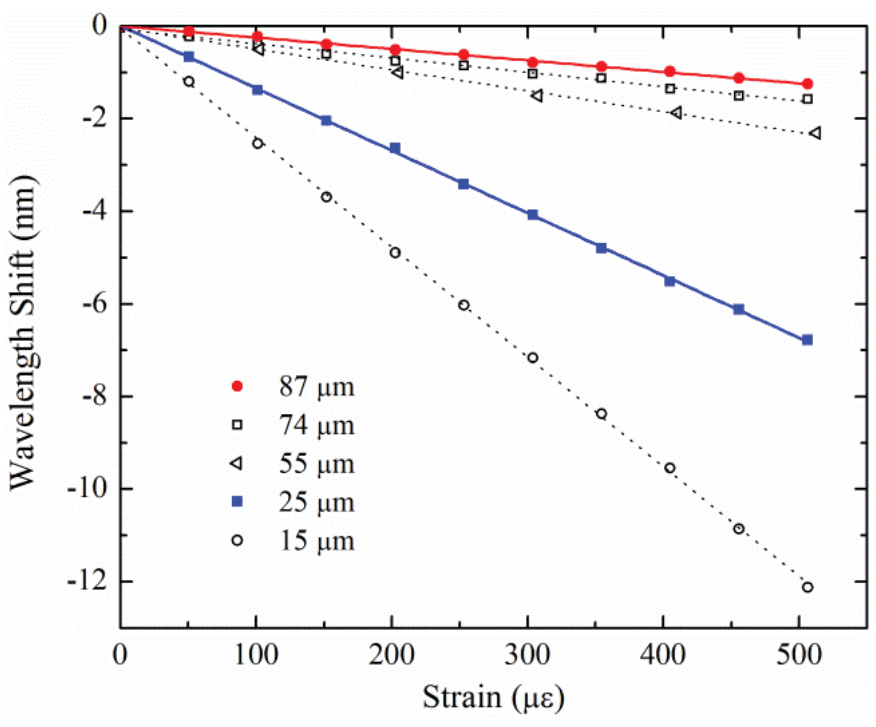

Fig. 4. Relationship between wavelength shift and applied strain for several taper structures. In full are the two sensing heads used in the simultaneous strain and temperature sensing (sensitivities in bold in Table I).

TABLE I

STRAIN SENSITIVITIES

\begin{tabular}{c|c}
\hline $\begin{array}{c}\text { Taper Diameter } \\
(\mu \mathrm{m})\end{array}$ & $\begin{array}{c}\text { Strain Sensitivity } \\
(\mathrm{pm} / \mu \varepsilon)\end{array}$ \\
\hline 125 & -2.08 \\
$\mathbf{8 7}$ & $-\mathbf{2 . 4 8}$ \\
74 & -3.09 \\
55 & -4.45 \\
$\mathbf{2 5}$ & $-\mathbf{1 3 . 5}$ \\
15 & -23.7 \\
\hline
\end{tabular}

If stress is applied to the taper structure, at constant temperature, the wavelength will be shifted by $\Delta \lambda_{\text {taper }}$ according to $\Delta \lambda_{\text {taper }}=\kappa_{0} \varepsilon_{\text {taper }}$. Where $\kappa_{0}$ is a constant characteristic of the fiber material, which can be easily determined experimentally by analyzing the variation of the wavelength as a function of strain, at constant temperature and $\varepsilon_{\text {taper }}$ is the strain applied to the taper region.

However, if strain is applied to the entire sensor, then an unequal load of stress will appear along each section of the sensor depending on the mechanical resistance and thus on the cross sectional area:

$$
\frac{\varepsilon_{\text {fiber }}}{\varepsilon_{\text {taper }}}=\frac{A_{\text {taper }}}{A_{\text {fiber }}}
$$

Combining this with the definition of strain, an expression for the wavelength shift is readily deduced:

$$
\Delta \lambda_{\text {taper }}=\kappa_{0} \frac{L_{\text {taper }}+L_{\text {fiber }}}{L_{\text {fiber }} \frac{d_{\text {taper }}^{2}}{d_{\text {fiber }}^{2}}+L_{\text {taper }}} \varepsilon_{0}
$$

where $L_{\text {taper }}$ and $L_{\text {fiber }}$ are $d_{\text {taper }}$ and $d_{\text {fiber }}$ are the diameters of taper and fiber respectively.

The theoretical expression plotted in Fig. 5 and given by equation (2) is derived from a purely geometrical point of view and is a mere estimate of the strain sensitivity and can be applied to a diversity of structures, namely FBGs [10]. 


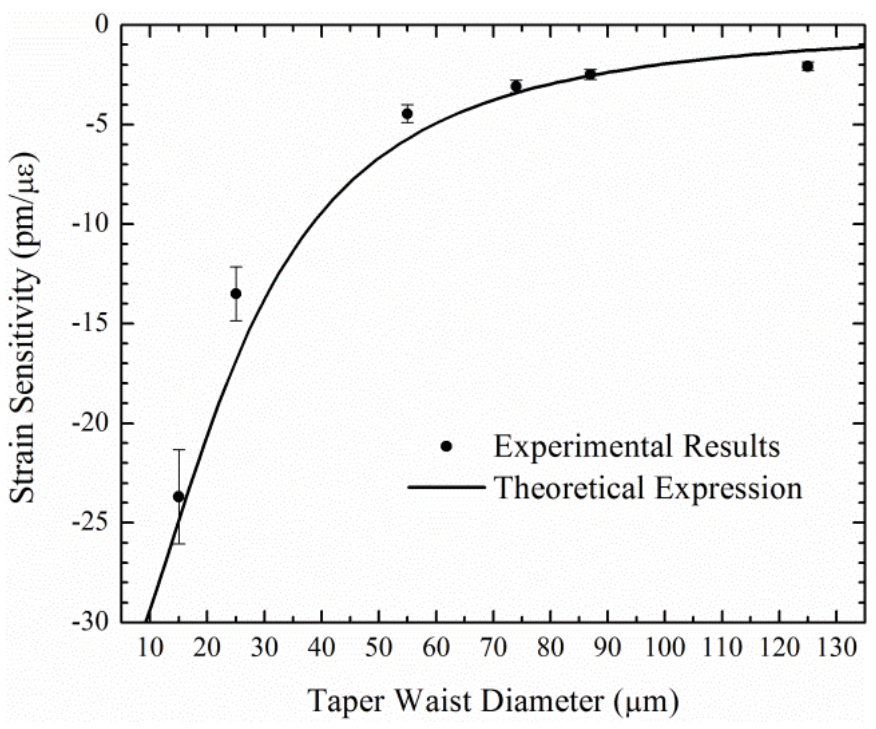

Fig. 5. Relationship between strain sensitivity and taper waist diameter. For the theoretical approximation, a value of $\kappa_{0}=-1.28 \mathrm{pm} / \mu \varepsilon$ was used.

Strain sensitivity varies nonlinearly with taper waist diameter. It increases as taper diameter decreases. Sensitivities of $-2.48 \mathrm{pm} / \mu \varepsilon$ and $-13.5 \mathrm{pm} / \mu \varepsilon$ were obtained for the $87 \mu \mathrm{m}$ and $25 \mu \mathrm{m}$ tapered SMSs. A tenfold increase in strain sensitivity was achieved when tapering the SMS structure from $125 \mu \mathrm{m}(-2.08 \mathrm{pm} / \mu \varepsilon)$ to $15 \mu \mathrm{m}(-23.69 \mathrm{pm} / \mu \varepsilon)$.

For temperature characterization, the sensing heads were heated in a cylindrical oven and the wavelength shift of the spectral response monitored (see Fig. 6). Different sensitivities were obtained for different sensing heads, namely $16.56 \mathrm{pm} /{ }^{\circ} \mathrm{C}$ for the $87 \mu \mathrm{m}$ taper and $13.95 \mathrm{pm} /{ }^{\circ} \mathrm{C}$ for the $25 \mu \mathrm{m}$ taper. The sensor's behavior to temperature is positive so the dominant parameter is thermal expansion.

These tapered SMS structures can be used for the simultaneous measurement of temperature and strain using two different diameter tapers. A solution is presented using the matrix method. Considering two different tapered SMS structures, namely the ones tapered down to $87 \mu \mathrm{m}$ and $25 \mu \mathrm{m}$ one has: $\Lambda=[\mathrm{K}] \Omega$ or,

$$
\left[\begin{array}{l}
\Delta \lambda_{(87)} \\
\Delta \lambda_{(25)}
\end{array}\right]=\left[\begin{array}{ll}
\kappa_{T(87)} & \kappa_{\varepsilon(87)} \\
\kappa_{T(25)} & \kappa_{\varepsilon(25)}
\end{array}\right]\left[\begin{array}{c}
\Delta T \\
\Delta \varepsilon
\end{array}\right]
$$

where $\kappa_{T}$ and $\kappa_{\varepsilon}$ are the sensitivities to temperature and strain.

Inverting the matrix expressions for the temperature and strain variations as a function of the sensitivities and wavelength shiftsone can obtain:

$$
\left[\begin{array}{c}
\Delta T \\
\Delta \varepsilon
\end{array}\right]=\frac{1}{D}\left[\begin{array}{cc}
\kappa_{\varepsilon(25)} & -\kappa_{\varepsilon(87)} \\
-\kappa_{T(25)} & \kappa_{T(87)}
\end{array}\right]\left[\begin{array}{l}
\Delta \lambda_{(87)} \\
\Delta \lambda_{(25)}
\end{array}\right]
$$

where $D=\kappa_{T(87)} \kappa_{\varepsilon(25)}-\kappa_{\varepsilon(87)} \kappa_{T(25)}$. The limitation of most methods reported for simultaneous measurement of pairs of quasi-static parameters arises from a small value of the matrix determinant, which makes it highly sensitive to noise. The configuration is substantially immune to this problem when the difference of the sensitivity slopes of the two SMS structures is large. From these values, given in Figs. 4 and 6,

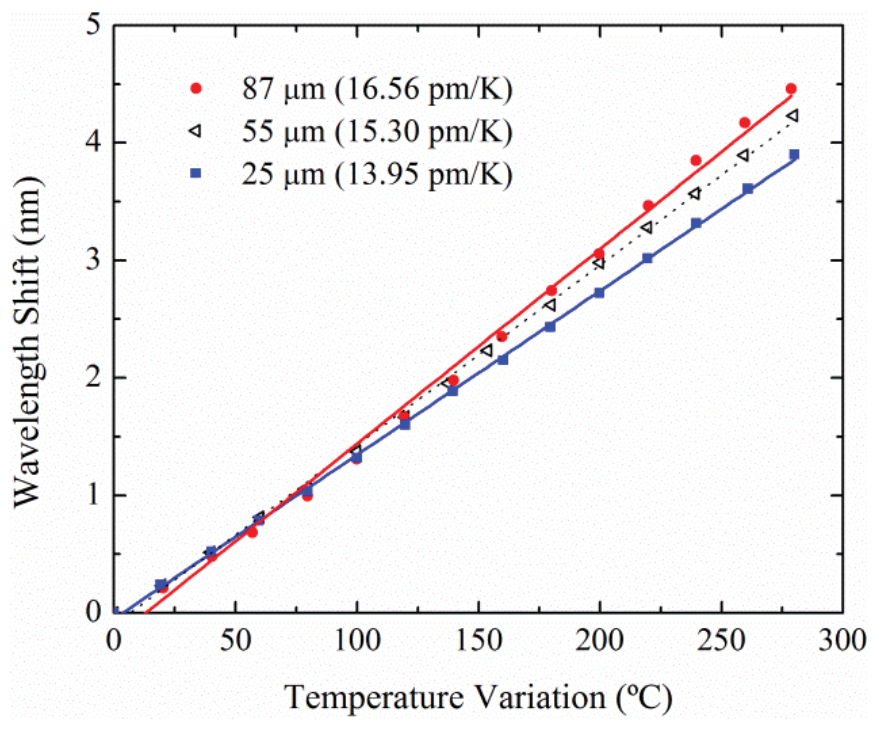

Fig. 6. Relationship between wavelength shift and temperature variation. In full are the two sensing heads used in the simultaneous strain and temperature sensing.

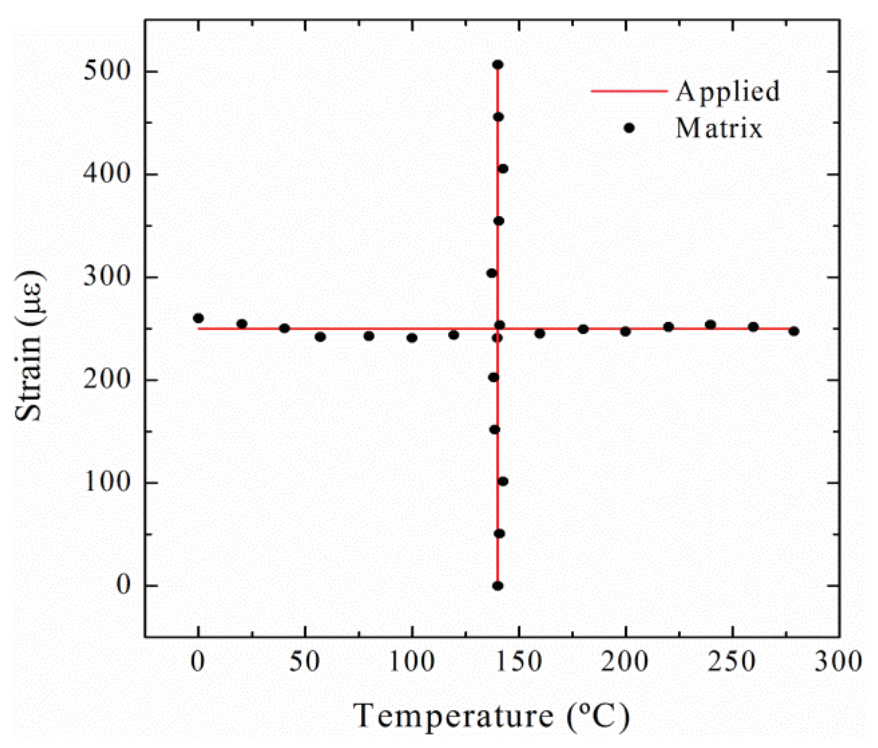

Fig. 7. Sensor output as determined by (5) for an applied strain at constant temperature and a temperature variation at constant strain.

equation (4) becomes:

$$
\left[\begin{array}{c}
\Delta T \\
\Delta \varepsilon
\end{array}\right]=-\frac{1}{189.0}\left[\begin{array}{cc}
-13.5 & 2.48 \\
-13.95 & 16.56
\end{array}\right]\left[\begin{array}{l}
\Delta \lambda_{(87)} \\
\Delta \lambda_{(25)}
\end{array}\right]
$$

The performance of this simultaneous measurement configuration was experimentally determined by undertaking strain variations in a range of $500 \mu \varepsilon$ at a fixed temperature $\left(140{ }^{\circ} \mathrm{C}\right)$ and the other way around, i.e., temperature variationsin a range of $280{ }^{\circ} \mathrm{C}$ for a specific applied strain $(\Delta \varepsilon=250 \mu \varepsilon)$. The results are expressed in Fig. 7, where maximum errors relative to the applied values appear. From these results, resolutions of $\pm 1.6{ }^{\circ} \mathrm{C}$ and $\pm 5.6 \mu \varepsilon$ were determined for temperature and strain measurements, respectively.

Besides determining the resolutions, it is necessary to determine the stability of the sensitivity matrix. Stability can be 
TABLE II

INVERSION MATRIX CONDITION NUMBER FOR SEVERAL SENSING CONFIGURATIONS

\begin{tabular}{c|cc}
\hline $\begin{array}{c}\text { Sensing } \\
\text { Configuration }\end{array}$ & $\begin{array}{c}\text { Condition } \\
\text { Number }\end{array}$ & $\begin{array}{c}\text { Reference } \\
\text { Number }\end{array}$ \\
\hline Tapered SMS structures & $\mathbf{3 . 1 6}$ & This work \\
FBG + Brillouin & 5.5 & {$[12]$} \\
FBG in MOF & 15.3 & {$[13]$} \\
Bragg Fibers & 21.3 & {$[14]$} \\
FBG in Taper & 26.4 & {$[15]$} \\
Tilted FBG & 39.4 & {$[16]$} \\
FBG + MMF & 45.0 & {$[17]$} \\
Type IA/IIA & 55.3 & {$[18]$} \\
\hline
\end{tabular}

verified through the condition number of the matrix, which is an algebraic quantity that provides a qualitative estimate for the sensitivity of the solution of a system of linear equations [11].

The larger the condition number of the matrix, the larger the upper bound for the relative error. Consequently, for matrices with high condition number, a small relative error in $[\Lambda]$ can lead to a large relative error in the solution $[\Omega]$. In order to have a well-conditioned system, cross-sensitivity between the two sensors is necessary, i.e., the one with higher temperature sensitivity must be the one with the lowest strain sensitivity. That is the case with the sensors considered. The condition number obtained is 3.16. It is interesting to determine the relative performance of several configurations using the matrices' condition number (see Table II). To the authors' knowledge, the sensing configuration presented has the lowest condition number when considering dual-parameter sensing of strain and temperature. The sensing configuration that gets the closest with a condition number of 5.5 monitors the wavelength of an FBG sensor and the frequency of the Brillouin gain spectra over a $20 \mathrm{~m}$ fiber length.

\section{CONCLUSION}

Tapers were produced on SMS structures and were characterized in strain and temperature. From the simulations it was ascertained that the creation of a taper in the MMF section of the SMS does not destroy the MMI pattern. In fact, what happens is that the self-images appear more closely packed in the taper region. Because of this, an increase of strain sensitivity was expected and confirmed since stretching the taper will lead to a greater shift in the MMI pattern and consequent change in output light field. Combining two different tapered SMS structures with different strain and temperature sensitivities it is possible to measure strain and temperature simultaneously. Resolutions of $\pm 1.6^{\circ} \mathrm{C}$ and $\pm 5.6 \mu \varepsilon$ were achieved.

\section{REFERENCES}

[1] L. Soldano and E. Pennings, "Optical multimode interference devices based on self-imaging: Principles and applications," J. Lightw. Technol., vol. 13, no. 4, pp. 615-627, Apr. 1995.

[2] A. Kumar, R. K. Varshney, S. Antony, and P. Sharma, "Transmission characteristics of SMS fiber optic sensor structures," Opt. Commun., vol. 219, no. 6, pp. 215-219, 2003.

[3] Q. Wang, G. Farrell, and W. Yan, "Investigation on single-modemultimode-single-mode fiber structure," J. Lightw. Technol., vol. 26, no. 5, pp. 512-519, Mar. 1, 2008.

[4] A. Mehta, W. Mohammed, and E. Johnson, "Multimode interferencebased fiber-optic displacement sensor," IEEE Photon. Technol. Lett., vol. 15, no. 8, pp. 1129-1131, Aug. 2003.

[5] W. Mohammed, A. Mehta, and E. Johnson, "Wavelength tunable fiber lens based on multimode interference," J. Lightw. Technol., vol. 22, no. 2, pp. 469-477, Feb. 2004.

[6] O. Frazäo, S. O. Silva, J. Viegas, L. A. Ferreira, F. M. Araújo, and J. L. Santos, "Optical fiber refractometry based on multimode interference," Appl. Opt., vol. 50, no. 25, pp. 184-188, Sep. 2011.

[7] W. S. Mohammed, P. W. E. Smith, and X. Gu, "All-fiber multimode interference bandpass filter," Opt. Lett., vol. 31, no. 17, pp. 2547-2549, Sep. 2006.

[8] Q. Wang and G. Farrell, "Multimode-fiber-based edge filter for optical wavelength measurement application and its design," Microw. Opt. Technol. Lett., vol. 48, no. 5, pp. 900-902, 2006.

[9] P. Wang, G. Brambilla, M. Ding, Y. Semenova, Q. Wu, and G. Farrell, "High-sensitivity, evanescent field refractometric sensor based on a tapered, multimode fiber interference," Opt. Lett., vol. 36, no. 12, pp. 2233-2235, Jun. 2011.

[10] O. Frazäo, S. F. O. Silva, A. Guerreiro, J. L. Santos, L. A. Ferreira, and F. M. Araújo, "Strain sensitivity control of fiber Bragg grating structures with fused tapers," Appl. Opt., vol. 46, no. 36, pp. 8578-8582, 2007.

[11] W. Jin, W. C. Michie, G. Thursby, M. Konstantaki, and B. Culshaw, "Simultaneous measurement of strain and temperature: Error analysis," Opt. Eng., vol. 36, pp. 598-609, Feb. 1997.

[12] M. Davis and A. Kersey, "Simultaneous measurement of temperature and strain using fibre Bragg gratings and brillouin scattering," IEE Proc. Optoelectron., vol. 144, no. 3, pp. 151-155, Jun. 1997.

[13] O. Frazäo, J. P. Carvalho, L. A. Ferreira, F. M. Araujo, and J. L. Santos, "Discrimination of strain and temperature using Bragg gratings in microstructured and standard optical fibres," Meas. Sci. Technol., vol. 16, no. 10, pp. 2109-2113, 2005.

[14] O. Frazäo, L. M. N. Amaral, J. M. Baptista, P. Roy, R. Jamier, and S. Février, "Strain and temperature discrimination using modal interferometry in Bragg fibers," IEEE Photon. Technol. Lett., vol. 22, no. 21, pp. 1616-1618, Nov. 1, 2010.

[15] O. Frazäo, M. Melo, P. V. S. Marques, and J. L. Santos, "Chirped Bragg grating fabricated in fused fibre taper for strain-temperature discrimination," Meas. Sci. Technol., vol. 16, no. 4, pp. 984-988, 2005

[16] E. Chehura, S. W. James, and R. P. Tatam, "Temperature and strain discrimination using a single tilted fibre Bragg grating," Opt. Commun., vol. 275, no. 2, pp. 344-347, 2007.

[17] D. P. Zhou, L. Wei, W. K. Liu, Y. Liu, and J. W. Y. Lit, "Simultaneous measurement for strain and temperature using fiber Bragg gratings and multimode fibers," Appl. Opt., vol. 47, no. 10, pp. 1668-1672, 2008.

[18] X. W. Shu, et al., "Dependence of temperature and strain coefficients on fiber grating type and its application to simultaneous temperature and strain measurement," Opt. Lett., vol. 27, no. 9, pp. 701-703, 2002. 